\title{
Téoros
}

Revue de recherche en tourisme

\section{Les parcs à thèmes. Essai de fondement sur une base naturelle}

\section{Jean-Claude Jay-Rayon et Brigitte Morneau}

Volume 12, numéro 3, octobre 1993

Le renouveau des parcs à thèmes

URI : https://id.erudit.org/iderudit/1077933ar

DOI : https://doi.org/10.7202/1077933ar

Aller au sommaire du numéro

Éditeur(s)

Université du Québec à Montréal

ISSN

0712-8657 (imprimé)

1923-2705 (numérique)

Découvrir la revue

Citer cet article

Jay-Rayon, J.-C. \& Morneau, B. (1993). Les parcs à thèmes. Essai de fondement sur une base naturelle. Téoros, 12(3), 18-21. https://doi.org/10.7202/1077933ar d'utilisation que vous pouvez consulter en ligne.

https://apropos.erudit.org/fr/usagers/politique-dutilisation/ 


\title{
Les parcs à thèmes. Essai de fondement sur une base naturelle
}

\author{
Jean-Claude Jay-Rayon et Brigitte Morneau*
}

Une des façons d'éclairer sous un certain angle plusieurs mécanismes propres aux parcs à thèmes, et d'essayer de mieux comprendre leurs multiples existences à succès, est de tenter d'en démonter des ensembles et de les traiter sur une base originale. C'est ce que nous avons tenté de faire en nous servant à la fois de l'Étbologie, de l'Écologie bumaine et de la Praxémique ${ }^{(1)}$ telles que développées par Konrad Lorenz, René Dubos, E.T. Hall et leurs écoles respectives.

Nous avons initié cette démarche de recherche et de prospective - significative mais fort modeste en soi - parce que nous estimons qu'étudier les parcs à thèmes sous l'angle exclusivement phénoménologique $=$ pour ce qu'ils coûtent et offrent aux clien tèles récréotouristiques - est insuffisant! Nous croyons que la compréhension d'un phénomène qui regroupe sous un même vocable, aussi bien les trois unités de Walt Disney World en Floride $e^{(2)}$, que La Ronde à Montréal ou, par extension, Old Sturbridge Village ${ }^{(j)}$ mérite qu'on s'attarde à ce qui se situe derrière la scène et l'expression populaire des thèmes.

De plus, s'attarder à énumérer tous les parcs à thèmes est aussi une tâche relativement laborieuse parce que d'une part, ils sont très nombreux et variés en Occident et particulièrement aux Etats-Unis. Et d'autre part, ils occupent beaucoup de champs d'intérêt, allant du simple domaine del'amusement forain et sensoriel, à celui hautement culturel relié à l'art et à l'histoire, en passant par des sujets scientifiques - ou encore - des $*$ mondes* imaginaires reconstitués et matérialisés.

Malgrécela, le liant unificateur qui semble les souder et les caractériser, c'est que tous ont développé un «unique sujetw

Monsieur Jean-Claude Jay. Rayon est concepteur. chercheur et maitre de conference et madame Brigitte Morneau est associee a la firme Tangram et s"occupe d'amenagement, de developpement et de formation en recréotourisme. connu de la plupart d'entre nous et qui nous habite comme être humain depuis la nuit des temps. Sujet qui, par la suite, a été transformé en infrastructures, en objets et en animation attrayante, de façon à constituer, sous des proportions agrandies ou colossales... des pares à thèmes.

\section{Derrière le thème culturel... I'élément bio-comportemental universel}

En fait, il est probable que ce n'est pas le thème lui-même qui détermine la réussite d'un... parc à thèmes, mais le fondement bio-comportemental qui lui sert d'assise. Par exemple, l'être humain étant porteur d'un imaginaire meublé d'animaux drôles ou apeurants et fantastiques et d'images environnementales fantasmagoriques qui surgissent dans les rêves ou les délires, il devenait alors normal que, la technique aidant, nous les construisions un jour dans la réalité d'un Magic Kingdom.

D'ailleurs, contrairement à ce que de nombreuses personnes croient, l'univers Disney élabore ses mondes et ses personnages à partir d'une base scientifique qui fait appel à l'ensemble de l'Étbologie bumaine. C'est ce que démontre en particulier Irenaüs Eibl-Eibesfeldt en traitant des mécanismes déclencheurs et des stimuli-clés chez l'homme ${ }^{(4)}$.

Il en est de même de la science, de la technologie et de la connaissance qui toutes font partie du *génies humain. En ce sens, le parc sciento-technologique d'Epcot Center à Orlando ou celui de Toronto, vont au-delà des simples jeux interactifs ou du spectacle. Ils présentent une partie de l'intelligence humaine même aux moins doués des visiteurs - et exposent un avenir prometteur dont ils pourront se servir matériellement et culturellement pour ne pas risquer de régresser à l'état de simple nature!
Quant aux parcs à thèmes zoologique, floristique, aquatique, biotique... des années 1995-2020, ceux-ci tendent déjà à matérialiser des sortes de *paradis perdus* où, hommes, bêtes et nature vivront en harmonie Proxémique. C'est là du moins l'image qui s'en dégage.

Le fait d'avoir déjà érigé technologiquement des portions d'océan en forme d'aquariums géants, de concevoir un «sensorium* par satellite pour le futur zoo de Londres, ou de reconstituer le biotopedans ledésert de l'Arizona ${ }^{(9)}$, nous indique que dorénavant on a l'intention de matérialiser le concept du sparadis terrestrew existant sous des formes différentes dans toutes les cultures. Là encore, le thème animal, végétal, biologique, naturel... n'est que la résurgence d'une baseculturelle, universelle et retranscrite.

Nous retrouvons aussi le même fondement derrière les parcs à thèmes historiques ou artistiques, tels les \&living museum maritimes, agraires, préhistoriques, qu'il ne faut surtout pas confondre avec certains décors récupérés après le tournage de séries télévisées.

Ces musées vivants qui reconstituent des époques entières s'appuient, là aussi, sur un besoin humain universel: la connaissance du temps historique transmis de mémoire, par le conte, la légende ou l'épopée fantastique et qui contribue à diminuer l'angoisse du futur et de la mort. Et c'est dans le même ordre d'idée que l'on pourrait envisager un parc à thèmes des religions, basé sur le concept religieux universel, un autre sur le hasard et le jeu et, pourquoi pas, un autre conçu à partir des représentations de la vie après la mort!

En somme, il semble probable que le parc à thèmes, s'il veut réussir à s'implanter et à mobiliser l'intérêt des foules devisiteurs, n'a d'autre choix que d'asseoir sa thématique sur un comportement fondamental, de préférence inné. Aussi surprenant que cela puisse paraître, le parc à thèmes - trop souvent considéré comme 
seulement divertissant, voire futile par certains - serait une représentation objective et moderne des rêves, fantasmes, croyances, mythologies, utopies, angoisses... qui habitent l'humanité entière. Ce qui expliquerait, du moins partiellement, leur facilité à s'inscrire dans un marché mondial du produit touristique.

En conséquence, se servir d'un thème quelconque et restreint pour bâtir un parc thématique, vouerait celui-ci à un quasi échec, compte tenu des ressources financières très importạntes qu'il faudrait pour le construire. A titre d'exemple simplifié, il serait parfaitement illusoire de vouloir fabriquer un parc à thèmes autour d'une seule pomme, même originelle! Il faudrait dans ce cas, pour avoir une chance de rếussir, bâtir la théma tique autour de toutes les pommes connues - et par extension, autour de tous les fruits comestibles - et ensuite asseoir le tout sur une animation qui reconstituerait le comportement de cueillette, qui, lui, enfin est fondamental.

\section{Au-delà du thème aussi... le gisement environnemental}

Le touriste - il s'agit d'une sorte d'évidence - est un individu qui, ayant volontairement abandonné son territoire familier, décide de se rendre ailleurs, dans un espace souvent étranger et éloigné. D'où ce réflexe de relative insécurité et la nécessité pour la majoritế d'entre nous d'occuper un site restreint à l'arrivée, de savoir préalablement quoi voir, où dormir, quoi manger, etc.

Quant aux touristes, *no wheres, ce sont ceux-là mểme qui, en général, étudient le mieux leurs itinéraires, les lieux d'arrêt, les moeurs rassurantes locales et la... gastronomie régionale pour revenir en santé! En résumé, le tourisme ed'aventure existe peu et le touriste en général se donne des balises mentales très précises en choisissant sa destination. A cela il faut ajouter que la durée des voyages allant en diminuant, on se rend de plus en plus loin avec un besoin d'efficacité accru quant à la consommation du produit touristique.

Dans ce contexte, les parcs à thèmes et aussi ceux dit naturels - dont le thème est la nature - répondent de plus en plus aux besoins du débutdu troisième millénaire.

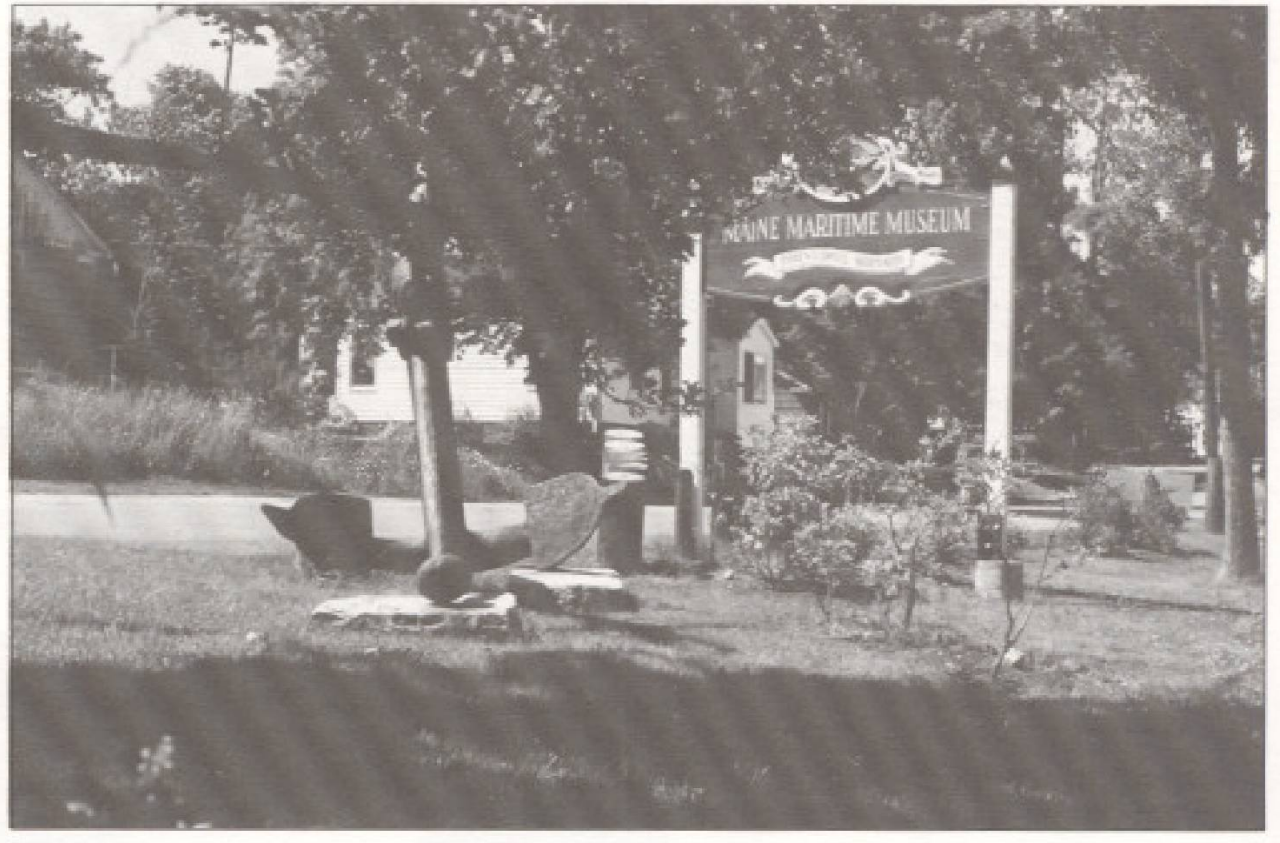

À des milliers de kilomètres du point de départ, ils concentrent sur un territoire minimum, le produit historique, sensoriel, artistique, culturel ou autre.

Dans cet ordre d'idée, si la majorité des parcs à thèmes se situent aux États-Unis, ce n'est pas sans raison! C'est parce que l'espace nord-américain ayant été considéré comme *vides par les Occidentaux qui l'occupèrent au début, il $y$ a encore une certaine nécessité d'en ponctuer l'espace global. Ce qui est totalement différenten Europe, en Asie ou en Afrique, où la plupart des attraits touristiques, issus de l'histoire millénaire, tiennent lieu de thèmes concentrés et d'endroits précis à visiter.

Donc, au-delà du thème proprement dit, le parc thématique représente, dans un endroit contraint, un produit à haute densité. Sans lui, il faudrait que le touriste explore à grand frais de temps et d'argent un pays ou un continent pour y découvrir autant d'élémentsattractifs. En somme, il constitue un gisement environnemental et agit comme un filon aurifêre dans une masse considérable de roches et de terre.

Et c'est à ce titre de gisement culturel et artistique comme à Brookgreen Gardens ${ }^{(6)}$ -de gisement historique maritime comme a Bath ${ }^{\text {() }}$ - de gisement muséal comme à Shelburne $e^{(\%)}$ - que le parc à thèmes correspond à un besoin existant depuis des millions d'années. Celui de l'efficacité de la destination pour se nourrir ou... se cultiver et se distraire.

En réalité, le comportement fondamental de l'homme considéré à partir de l'Écologie et de l'Étbologie bumaine, nous indique qu'on ne s'est jamais dispersé naturellement dans l'espace, sauf en cas de danger ponctuel et de fuite momentanée. Ce simple principe devrait d'ailleurs inspirer les futurs schémas d'aménagement des MRC au plan de l'aménagement touristique et corriger certaines errances en matière d'utilisation de l'espace.

D'il y a quatre ou cinq millions d'années à nos jours - de l'bomo babilisà aujourd'hui - nous avons toujours préféré des cibles concentrées pour nous approvisionner en cueillant, en chassant, en pêchant, pour nous abreuver et maintenant... pour nous divertir. Dans ce contexte, il est alors probable que le parc à thèmes réponde encore pendant longtemps à ce bio-comportement enfoui dans la mémoire génétique de notre espèce.

À cet effet, René Dubos dans son volume intitulé Choisir d'être humain (1974) précisait qu' $*[. .$.$] en dépit des apparences,$ I'humanité est presque toujours fondamentalement sédentaire- - même et surtout après avoir parcouru les milliers de kilomètres linéaires pour atteindre une destination touristique. Et il ajoutait: 


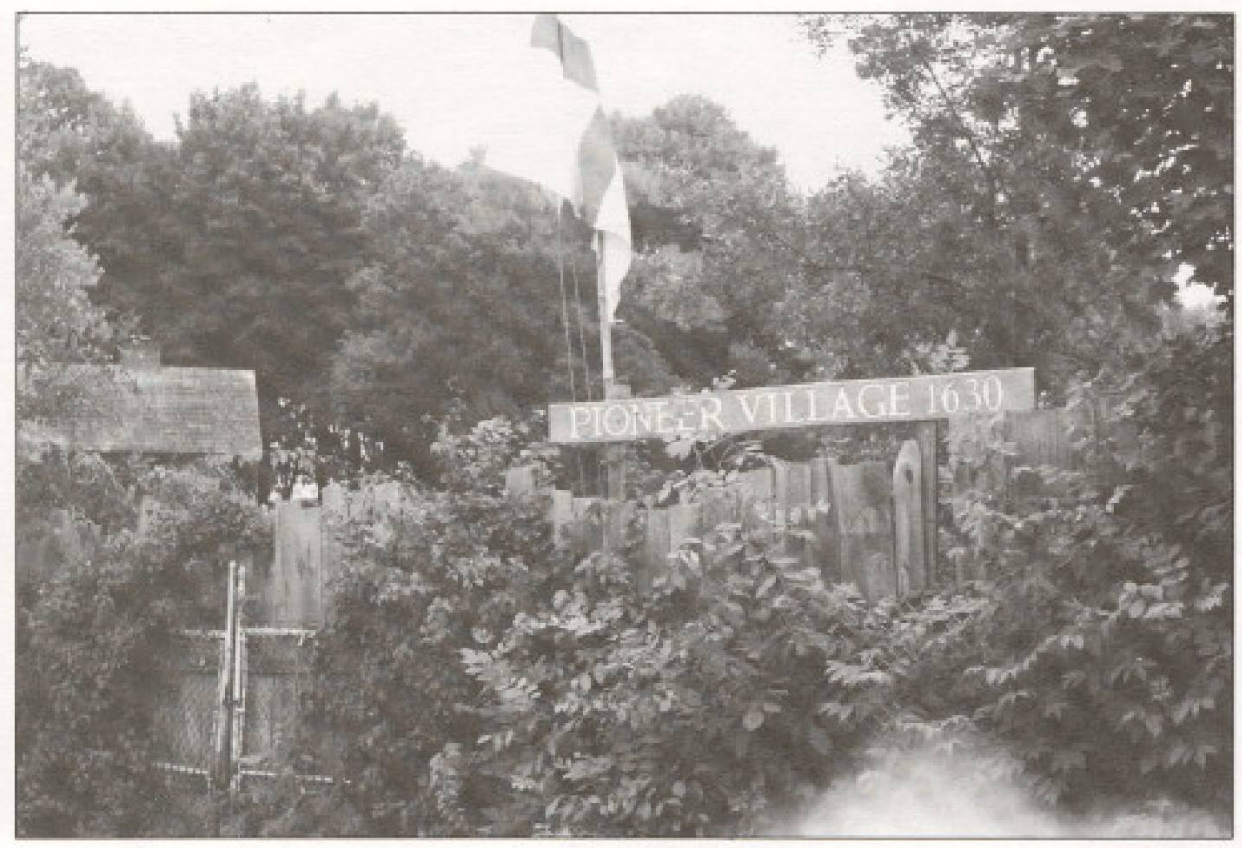

*[...] nous ne fonctionnons bien et confor tablement que dans un milieu dont nous connaissons les ressources et les dangers. Même à l'heure actuelle, l'immense majorité des humains vivent et meurent dans un rayon de quelques kilomètress (Le camp et la route, p. 135).

Dans cet esprit, non seulement les parcs à thèmes sont là pour rester mais il y a fort à parier que de nouveaux surgiront et qu'ils tendront graduellement à corriger la dispersion actuelle du produit touristique.

Mieux encore! Certains parcs thématiques du futur - 2010-2020 - risquent de nous surprendre par le fait qu'ils correspondront de plus en plus, avec l'aide d'une technologie et d'une informatisation avancée, à la nature fondamentale de l'être humain. Car, il faudra bien en convenir tôt ou tard, Magic Kingdom - en apparence totalement artificiel - est plus proche de l'Exologie bumaine sous certains aspects que bien des parcs naturels à dominante végétale, animale ou minérale. Cela peut surprendre, mais il en est ainsi et Mickey Mouse, tout en étant fait de plastique et de fibres synthétiques, déclenche, par ses formes enfantines, une sympathie naturelle reliée à la mémoire génétique des millions de personnes qui lui rendent visite ${ }^{9}$.

\section{Consommer... oui, à profusion!}

Ajouté aux deux éléments précédemment développés, il s'en trouve un troisième qui, lui concerne le rassemblement humain et la consommation. Reconnaissons-le sans ambage ni culpabilité, se rendre dans un parc à thèmes, peu importe ce qui s'y trouve, c'est mettre les pieds dans l'univers de l'hyperchoix où chaque instant est appelé à stimuler la clientèle. A un tel point d'ailleurs, que certains le même billet pour deux jours de visite, estimant que le produit offert ne peut être consommé en une journée tellement il est... *copieux».

Là aussi, pour bien saisir ce phénomène dans sa réalitế, il faut $\mathrm{y}$ introduire des considérants qui remontent à notre héritage comportemental naturel. En ce sens, l'humain, comme d'ailleurs presque tous les êtres vivants sociaux, ont développé un comportement de survie et... de fête, en occupant des espaces qui contenaient le plus d'éléments spécifiques à consommer. D'abord, dans un but de simple alimentation, copulation ou grégarisme puis, par extension, pour toutes les formes de consommation possibles.

En conséquence, la rentabilité d'un parc à thèmes repose essentiellement sur un principe de profusion et de concentration de la consommation sur un même site. Et en ce sens, le parc à thèmes doit d'entre eux, culturels entre autre, offrent non seulement satisfaire le visiteur dans le temps présent, mais lui permettre d'emmagasiner une somme énorme de données pour alimenter ses souvenirs longtemps après son séjour. À cet effet, la densité et la cohérence des produits dérivés sont alors une des clés du succès ou del'échec financier d'un parcà thèmes ${ }^{(10)}$.

En allant un peu plus loin au niveau des déclencheurs, nous dirons que le rassemblement de multiples éléments similaires mais non identiques dans un même endroit, sous le même thème, constitue une unité perceptuelle gigantesque et *rentables au plan bio-comportemen$\mathrm{tal}^{(\mathrm{ili})}$.

À ce titre, il s'agit d'assimiler le fait que, pour la perception et le décodage environnemental, les centaines de milliers d'oies de Cap Tourmente deviennent... une seule oie gigantesque - les dizaines de châteaux de la Loire... un seul et immense palais - les centaines de sculptures de la Caroline du Nord... un seul et colossal monument, etc.

En ce sens, le parc à thèmes bien construit, par un effet de systémie, crée une attraction avec laquelle il est difficile de rivaliser dans la mise en marché. Ál'autre bout de cette perception du client, l'objet rare et unique, peut concurrencer cet effet d'entraînement.

En somme, ce qu'il faut retenir, c'est que le parcà thèmes, au-delà del'organisation systématique de ses parties, agit naturellement au niveau attractif comme le fait l'éléphant dans un zoo parmi les animaux plus petits - la baleine dans l'océan, parmi les poissons communs - ou l'Empire State Building parmi les habitations de New York. Il représente le grossissement et la réussite d'une idée poussée à son paroxysme et la notion de eprogrès» où, seul le ciel devient la limite de la volonté humaine ${ }^{(12)}$.

\section{Conclusion}

À notre avis, et considérés sur une base technico-scientifique, non seulement les pares à thèmes sont là pour proliférer mais aussi, pour s'agrandir et se renforcer à partir de nouvelles thématiques originales. Il est probable qu'ils dépasseront à brève échéance, si ce n'est déjà fait, la simple notion de... parc et s'étendront à 
celle d'endroits ou même de villes à thèmes.

Le succès des médiévales de 1993 à Québec; ville qui n'a rien à voir avec l'époque et l'histoire moyenâgeuse, en est un exemple flagrant. Pares, endroits, environnements, saisons et régions à thèmes tendront de plus en plus à spécifier le produit touristique international et à le rationaliser dans le temps et l'espace, vouant le concept de dispersion des attraits touristiques à une époque de plus en plus révolue.

Toutefois, pour que les thèmes réussissent à s'implanter et à créer une économie et une rentabilité elle-même génératrice d'emplois et d'entreprises, il s'agira de les considérer comme le résultat de soigneuses recherches appliquées, et non comme une résurgence de la *pensée magique».

Aussi étrange et ambigu que cela puisse paraître à priori, il nous faudra, pour occuper ce champ, «rebâtir» sur une nouvelle base plus sûre, et surtout moins émotive, notre perception actuelle de l'écologie humaine tout en la situant résolument dans un contexte prospectif. Celui de la techno-nature.

Reconnaissons-le sans complaisance, beaucoup de parcs à thèmes, dont les principaux sont américains de conception, sont encore dénigrés par certains intellectuels et mouvements écologistes et abordés sous l'angle d'univers artificiels... anti-naturels!' Or, nous estimons que cette perception négative empêche d'aborder le sujet pour ce qu'il est profondément, peu importe les idéologies qui le recouvrent temporairement. $\mathrm{Ce}$ qui nuit souvent à sa connaissance et à la réalité des faits!(13) $f$

\section{Notes}

(1) Ethologie, Écologie humaine et Proxémique: disciplines scientifiques traitant du comportement biologique, inne, de l'habitat de l'homme et des rapports de la personne a l'espace.

(2) Magic Kingdom - Epcot Center et MGM Studio. Trois parcs a thèmes parmi les plus connus au monde.

(3) Musée vivant base sur le XVIII" et XIX" siècle et qui fait suite aux époques précédentes représentés par ceux de Plymouth Plantation et de Salem.

(4) Éthologie, biologie du comportement, 1964, pp. 568.582 .

(5) Biosphère II, dont l'expérience humaine d'isolement vient de se terminer apres deux ans, en octobre 1993.

(6) Ancienne plantation transtormbe en jardin floral et shabitée de plusieurs centaines de sculptures en Caroline du Nord.

(7) Musbe vivant maritime situé dans le Maine

(8) Musée constitué de dizaines de sous-musées et abritant des collections uniques au monde - Vermont.

(9) Têtegrande par rapport au corps - front bombépar rapport a la dimension du reste de la face - veux trés grands situess au-dessous de la moitie de la face -extrémilés courtes et potelées - corps de forme ronde - surfaces du corps molles of blasti. ques - joues rondes protuberantes, etc.

(10) Le parc à thèmes est non seulement à considéfer sur le plan des infrastructures et des services. mais surtout au niveau manufacturier des souvenirs qui sont les produits dérivés à partir du thème central.

(11) Les centaines de personnages de Walt Disney, similaires mais... differents - les centaines de sculptures de Brookgreen Gardens apparentées mais... différenciées, les milliers d'oies en migration semblables mais... différentes, etc.

(12) Cet effet attractif du plus gros et du plus fort se retrouve aussi chez le chasseur et pecheur par rapport au trophee. Toutefos, il origine d'un réflexe de protection étudié à partir des bancs de poissons, des troupeaux de mammiferes et... des foules humaines.

(13) Il est malheureusement de bonton dans plusieurs milieux de denigrer encore ce concept du parc a thèmes. Le dernier débat sur ce sujet s'est déroulé entre les développeurs de la Chute Montmorency à Quebecet un groupe d'bcologistes, au printemps 1990.

\section{Bibliographie restreinte}

BLUMENSCHINE, Robert et John CAVALLO, Nos ancétres, des charognards? : Pour la science, no 182. décembre 1992.

Dubos, Rene, Choisir d'atre humain, notre terre ot son avenir, Edditions Denoeil, 1974, 207 p.

Dubos, Fené, Courtison la terre, Editions Stock, 1990. $243 \mathrm{p}$.
Eibl-Eibesteldt, Irenäus, Ethologie, blologle du com portement, nouvelle edition, Editions scientifiques Naturalia et Biologia, Paris, 1984, 748 p.

Jay-Rayon, Jean-Claude et Brigitte Morneau, * Prospective locale et regionale, 1995-2010: scénarios tendanciels et exploratoiress, T6oros, vol. 12, juillet 1993, pp. 4451.

Lorenz, Konrad, L'envere du mirolr, une histoire naturelle da la connaissance, Flammarion, France, $1975,342 \mathrm{p}$.

NINIO, Jacques, L'empreinte desene, Editions Jacob, $1969,304 \mathrm{p}$. 